\title{
INTRAORAL X-RAYS TO SUIT YOUR PRACTICE
}

Since January 2014 Sirona has been supplying users of the intraoral X-ray tube HELIODENTPLUS with a mobile stand at their request. This means that the device is always within reach and can be adapted to suit the individual requirements of every practice.

As well as being able to attach the X-ray tube on the wall or ceiling, HELIODENTPLUS users can now also attach it to a mobile stand. This flexibility not only improves the daily workflow, but also makes X-rays more comfortable for patients. This assembly option is particularly suitable for clinics and nursing homes so even if patients are immobile, X-rays that are necessary for treatment can still be taken without having to move the patient to a practice. In large practices the mobile stand allows HELIODENTPLUS to be quickly and easily moved from room to room. The scope of delivery also includes a tray that can be assembled if required, offering space for a laptop, documents and an XIOS XG intraoral sensor system.

Top image quality with the lowest radiation dose is extremely important at Sirona when it comes to intraoral X-rays. Sirona devices stand out because of their durability and are designed to handle a variety of requirements so that every dental practice will find the X-ray equipment to suit their needs.

For further information 08450715040 or email info@sironadental.co.uk.

\section{SCAN THE BAR CODE OF DENTAL IMPLANTS}

iChiropro from Bien-Air is now supplied with the Apple lightening connection as standard as well as the iPad Air Adaptor and iPad 4 adaptor to make your working life even easier.

iPad 4 and iPad Air have the higher resolution camera, which enables users to scan the implant bar code of most implants. The intuitive bar code scanner is just one of the many innovative features of the iChiropro App.

The iChiropro by Bien-Air is a revolutionary physiodispenser, controlled from an iPad. Simple to use, the intuitive, ergonomic application ensures significant time savings during operations. Available free from the Apple App Store, the iChiropro application opens up numerous avenues in terms of customisation options. New innovative functions are added approximately every 6-10 months, to guarantee users a simple, ergonomic and up-to-date experience.
The multi-user iChiropro incorporates the complete sequences of the leading implant manufacturers with their predefined settings. It also offers the option of saving and exporting operation data, in the form of graphics and tables, attached to the patient file built into the control interface. It's the only device which allows users to save, export and print all operation parameters, whilst documenting these with the patient data and the implants used. The application allows multiple users to customise and store their own sequences.

For more details or an insurgery demonstration, telephone 01293550200 or email ba-uk@ bienair.com.

\section{EASY CLOUD STORAGE FOR} YOUR X-RAYS AND IMAGES

cephX is an easy to use, cloudbased system that provides orthodontists and dentists with accurate cephalometric analyses and convenient image storage.

Analyses, photos, X-rays and worksheets are available 24/7, simply and easily from any location and in real-time, using a secure log-in. The cephX system is ideal for multiple practices, home access and optional consultation.

As documents are available to you via the cloud from anywhere, there's no need to install costly hardware or software to use cephX.

Ceph tracing is generally a tedious and time consuming process. cephX provides you with a complete solution that gives you the accurate analyses you need without the hassle. Simply upload a new ceph to your account and within two business days you will be able to view, edit and print all standard cephalometric analyses directly from the cloud. cephX also allows you to request custom analyses beyond the 60 standardly offered.

cephX allows you to create and store a variety of orthodontic treatment plans and record charts which can all be easily accessed and viewed via your cephX account. cephX also allows you to share data and consult with other dentists - even if they aren't registered users.

To find out more about cephX visit www.cephx.com. 